\title{
Computational Tools for the Investigation of the Male Lower Urinary Tract Functionality in Health and Disease
}

\author{
Chiara Giulia Fontanella ${ }^{1,2} \cdot$ Emanuele Luigi Carniel $^{1,2} \mathbb{D}$
}

Received: 31 August 2020 / Accepted: 16 January 2021 / Published online: 1 March 2021

(c) The Author(s) 2021

\begin{abstract}
Purpose This paper aims to show the potentialities of computational bioengineering in the field of lower urinary tract pathophysiology. Engineering methods allow the investigation of urine flow in healthy and pathologic conditions and the analysis of urethral occlusion by means of artificial urinary sphincters.

Methods Computational models of bladder and urethra were developed and exploited to investigate the lower urinary tract physiology in health and in disease. Average male morphometric configurations were assumed, together with typical properties of both biological tissues and fluids. The reliability of the models was assessed by the mutual comparison of results and the investigation of data from experimental and clinical activities.

Results The developed models allowed to analyze typical situations, such as the micturition in health and in disease, and the lumen occlusion by external devices. The models provided information that clinical and experimental tests barely provide, as the occurrence of turbulent phenomena within urine flow, the shear stresses at the lumen wall, the external pressure that is strictly required to occlude the lumen.

Conclusions The methods of bioengineering allow broadening and deepening the knowledge of the lower urinary tract functionality. More in detail, modeling techniques provide information that contributes to explain the occurrence of pathological situations, and allows to design and to optimize clinical-surgical procedures and devices.
\end{abstract}

Keywords Lower urinary tract · Fluid dynamics $\cdot$ Structural mechanics $\cdot$ Fluid structure interaction $\cdot$ Computational methods

\section{Abbreviations \\ CFD Computational fluid dynamics \\ CSM Computational structural mechanics \\ FSI Fluid structure interaction \\ AUS Artificial urinary sphincter}

\section{Introduction}

The comprehension of lower urinary tract physiology in health and in disease requires the investigation of urethral mechanical functionality [1-4]. The analysis pertains to the framework of biomechanics and provides considerable

Emanuele Luigi Carniel

emanueleluigi.carniel@unipd.it

1 Department of Industrial Engineering, University of Padova, Via Venezia 1, 35131 Padova, Italy

2 Centre for Mechanics of Biological Materials, University of Padova, Via F. Marzolo 9, 35131 Padova, Italy complexity, because it couples both solid and fluid mechanics problems [5, 6]. Bladder and urethra are among the principal components of the lower urinary tract. The bladder is a hollow and distensible viscus, and its wall is a complex structure made up of mucosa, submucosa, muscolaris and serosa layers. The male urethra is a hollow tube, which is composed by different regions, such as prostatic, membranous, bulbar and penile urethra [7]. The cross-sectional shape of the urethral lumen ranges between slit- and starlike ones in the absence of urine flow, while it assumes an almost circular shape during micturition $[8,9]$. The urethral wall is composed of an inner lamina propria, such as a mucosa-submucosa layer, while an outer muscular layer characterizes the prostatic and the membranous urethra, and a thick spongy layer defines the outer shell of both the bulbar and the penile urethra [10]. The natural urethral sphincter is located all around the prostatic and the membranous urethra.

The analyses of urine flow and pressure along the urethra are typical tests that are performed to investigate how the bladder and the urethral sphincter are working, to discover 
obstructions or to diagnose patients who have urinary incontinence or other symptoms and diseases, such as prostatic hyperplasia, reflux of urine in the prostatic ducts, prostatitis and dysuria $[7,11,12]$. The accurate knowledge of the pattern of urine fluid dynamics from the bladder up to the external meatus should provide a reliable tool for a better comprehension and explanation of results from uroflowmetry and urodynamic tests. Furthermore, such clinical investigations are usually invasive and painful. Computational Fluid Dynamics (CFD) [13] provides quantitative data about the urine flow regime depending on many different influencing factors, such as the specific morphometry of the lower urinary tract and obstruction phenomena [14-17]. The development of CFD models requires the acquisition of morphometric data by means of non-invasive techniques, such as echography or MRI. In detail, fluid dynamics analyses provide data about urine pressure, velocity and turbulence. The latter frequently entails inflammation of urethral mucosa, as well as stagnation and proliferation of bacteria, which are among the principal causes of dysuria $[18,19]$.

Moreover, urethra is not a rigid tube, but soft biological tissues build up the urethral wall providing for a considerably compliant duct. Urethral wall dilatation because of urine pressure should be analyzed [20,21]. Computational Solid Mechanics (CSM) [22] provides tools for the investigation of such dilatation phenomena, together with data about stress and strain fields within urethral wall [23]. The deformation of the urethra, in turn, affects the urine flow regime, entailing the coupling of fluid flow and solid mechanics problems, which is the framework of the so-called Fluid Structure Interaction (FSI) phenomena [5, 24]. Nowadays, many different applications in the cardiovascular field have proven the strength and the reliability of the FSI approach [25].

A further field of investigation for CSM and FSI pertains to urinary incontinence, which is one of the principal health concerns worldwide. Despite the Artificial Urinary Sphincter (AUS) is the gold standard for the treatment of male incontinence [26], up to $50 \%$ of patients receiving AUS requires surgical revision after initial placement [27-30]. Device malfunction, urethral atrophy, urethral erosion or infection processes mainly determine AUS failure. AUS application usually occurs all around the bulbar urethra, which is a non sphincteric region, where the local tissues are not used to support occluding actions. AUS has to ensure continence up to the highest bladder pressure conditions, and consequently it constantly applies a tiring occluding action. Such mechanical stimulation of urethral tissues is responsible for damage phenomena that may evolve to atrophy or erosion [27]. CSM and FSI allow analyzing mechanical interaction phenomena between AUS and urethra and provide quantitative information about AUS mechanical reliability. More in detail, the computational methods provide information about stress and strain within ureteral tissues, which are responsible for many different mechano-biological phenomena, such as tissue damage and vasoconstriction. In this sense, the results from computational investigations are mandatory for the optimal design of novel and more reliably AUS devices [31-34].

Previous studies reported about the computational investigation of lower urinary tract functionality, by means of a specific technique only, with particular regard to CFD methods [14-17]. The present work envisages the integration and the coupling of different computational techniques, showing the potentialities and the limitations of the different approaches. The activities aim to highlight the potentialities of computational methods of CFD, CSM and FSI for the investigation of lower urinary tract functionality in health and in disease. The different simulation scenarios are summarized in Table 1.

\section{Materials and Methods}

In the last decades, the methods of engineering burst into the field of medicine and biology and provided innovative tools for the investigation of functionality of biological systems, the reliability assessment, the planning and the design of procedures and devices. The engineering approach is usually based on the definition of a physical model of the investigated system by means of a coupled experimental and computational approach. Experimental activities are mandatory for model definition, identification and validation. With particular regard to the

Table 1 Summary of the different computational techniques, with applications, potentialities and advantages

\begin{tabular}{|c|c|c|}
\hline Computational methods & Applications & Potentialities and advantages \\
\hline Computational fluid mechanics (CFD) & Fluid flow along bladder and urethra & $\begin{array}{l}\text { Comprehensive evaluation of flow properties along } \\
\text { the entire urethra } \\
\text { Low computational effort }\end{array}$ \\
\hline Computational structural mechanics (CSM) & Urethral lumen occlusion by external actions & $\begin{array}{l}\text { Evaluation of relationships between occluding } \\
\text { action, intraluminal pressure and lumen area } \\
\text { Low computational effort }\end{array}$ \\
\hline Fluid structure interaction (FSI) & $\begin{array}{l}\text { Fluid flow along bulbar urethra } \\
\text { Urethral lumen occlusion by external actions }\end{array}$ & $\begin{array}{l}\text { Actual evaluation of urethral behavior, considering } \\
\text { the influence of both wall mechanics and urine } \\
\text { flow } \\
\text { High computational effort }\end{array}$ \\
\hline
\end{tabular}


fields of solid mechanics and fluid-dynamics, experimentations must provide data about the geometrical configuration of the biological district, the mechanical behavior of the building tissues and of the involved fluids. In this sense, notes about lower urinary tract histo-morphometry and mechanical experimentations are reported in Sect. 2.1. Computational methods allow to broad experimental results to an extremely wider scenario, considering many different configurations of the system, as many different patient situations, and many different loading conditions, considering also surgical procedures and devices. Furthermore, computational models provide information that barely can be achieved by experimental activities, as the stress and the strain fields, the fluid velocity and turbulence, etc. The potentialities of different computational methods, as CFD, CSM and FSI are consequently reported in sections from 2.2 to 2.5. Stress and strain provide relevant information in the field of tissue mechanics, because they are responsible for tissue damage and failure, tissue adaptation and mechano-transduction phenomena. On the other hand, the precise prediction of the flow within biological ducts is mandatory to evaluate the effects of pathologies, or to investigate the consequences of fluid actions on the duct wall.

\subsection{Experimental Characterization of the Male Lower Urinary Tract}

The geometrical configuration of the male lower urinary tract was obtained by the investigation of bladder and urethra histoanatomical configurations. Average data obtained by bladder and urethra morphometry and histology [7-10] made it possible to develop 3D CAD models of the lower urinary tract (UGS NX, Siemens PLM Software, Plano, Texas, USA).

The mechanical behavior of both bladder and urethra walls is usually investigated by means of experimentations performed on tissue samples from animal models, human cadavers and surgical residuals. The tissues mechanical response has been mostly investigated via uni-axial $[20,35]$ and bi-axial [36] tensile tests. The mechanical behavior of the entire biological structure is usually analyzed by means of insufflation tests $[20,37,38]$. The tissues microstructural changes occurring during stretching (i.e.: collagen fibers uncramping and alignment) induce a progressive increase of material stiffness, which entails the non-linearity of the stress-strain response. This is a typical phenomenon of soft biological tissues mechanics, which is usually interpreted by means of hyperelastic or viscohyperelastic constitutive formulations [23].

\subsection{CFD Analysis of Micturition}

Computational Fluid Dynamics allows to simulate and to analyze the flow of urine from the bladder up to the external meatus. The bladder and the urethra inner surfaces define the mathematical domain of urine flow and, in the framework of CFD, they are assumed to be not deformable. The average three-dimensional model of the urine domain was defined by the analysis of the bladder and urethra morphometry (Fig. 1a): an ellipsoidal conformation was assumed for the bladder, while the urethra was defined by means of a circular tube with variable diameter (prostatic region: $25 \mathrm{~mm}$ length, $1.9 \mathrm{~mm}$ radius; membranous region: $15 \mathrm{~mm}$ length, $1.9 \mathrm{~mm}$ radius; bulbar region: $40 \mathrm{~mm}$ length, $2.0 \mathrm{~mm}$ radius; penile region: $110 \mathrm{~mm}$ length, $1.5 \mathrm{~mm}$ radius; fossa navicularis: $1.8 \mathrm{~mm}$ radius; meatus: $1.2 \mathrm{~mm}$ radius). The urine domain was discretized by means of hexahedral elements within the boundary layer and tetrahedral elements elsewhere. The final mesh consisted of about 2,840,000 elements, considering different mesh sizes in order to provide a reliable discretization and a reasonable computational effort. No slip condition characterized the interaction between urine and wall domain. The classical Navier-Stokes equations defined the mathematical problem, together with the $\mathrm{k}-\varepsilon$ model for the description of turbulent flow mean conditions [13]. The material characterization of urine assumed incompressible and Newtonian behavior. Density and viscosity at $37{ }^{\circ} \mathrm{C}$ were identified on the basis of data from Inman et al. (2013) [39], as $1.02 \mathrm{~kg} / \mathrm{m}^{3}$ and $0.83 \mathrm{cSt}$, respectively. The boundary conditions of the CFD problem aimed at analyzing the typical micturition, characterized by a bladder wall pressure (inlet pressure condition) and atmospheric pressure at the end of the urinary meatus (outlet pressure condition) (Fig. 1a). The bladder pressure includes the contributions from both detrusor and abdominal pressure and ranges between 0 and $12 \mathrm{kPa}$ [40-43]. Transient CFD simulations were performed: the inlet bladder pressure condition was increased from 0 up to the target value in $2 \mathrm{~s}$, subsequently the bladder pressure was kept constant for $5 \mathrm{~s}$ aiming to achieve an almost steady state flow situation.

Aiming to further exploit the CFD approach to the investigation of the lower urinary tract functionality, modifications of the urethra model geometry allowed to analyze different stricture scenarios. Urethral stricture was applied in the bulbar region to simulate typical clinical situations. Different stricture seizes were investigated, such as $40 \%, 55 \%$ and $70 \%$ obstruction conditions.

CFD simulations have been performed by means of the general purpose code Comsol Multiphysics 5.4 (Comsol Inc., Burlington, MA, USA).

\subsection{FSI Analysis of Micturition}

The CFD approach to the investigation of micturition assumes rigid body behavior of both bladder and urethra walls. However, urine flow depends on the geometrical configuration of bladder and urethra domains, while the urine pressure induces dilatation phenomena of bladder and urethra walls, leading to modifications of the fluid domain. The interaction between the 

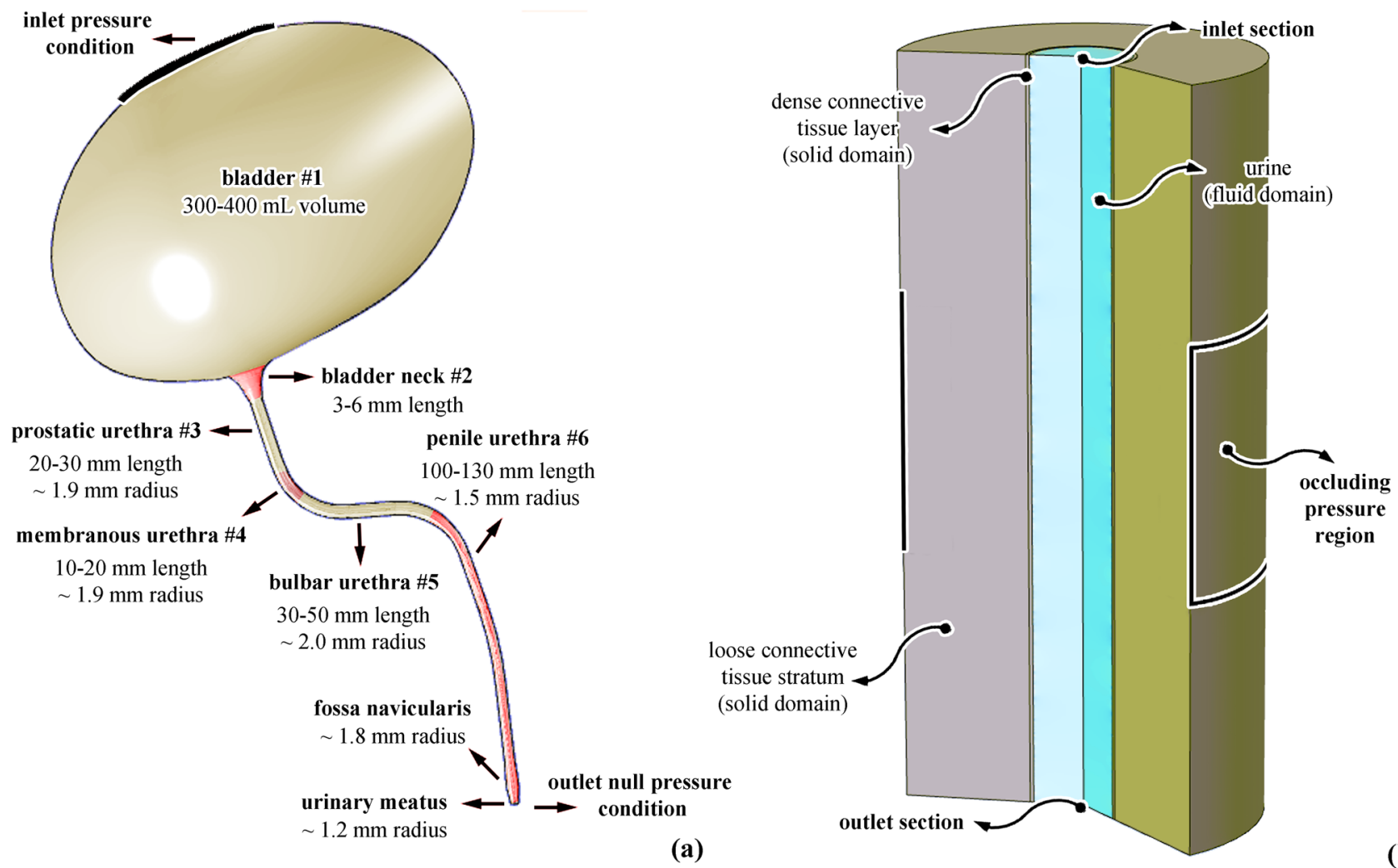

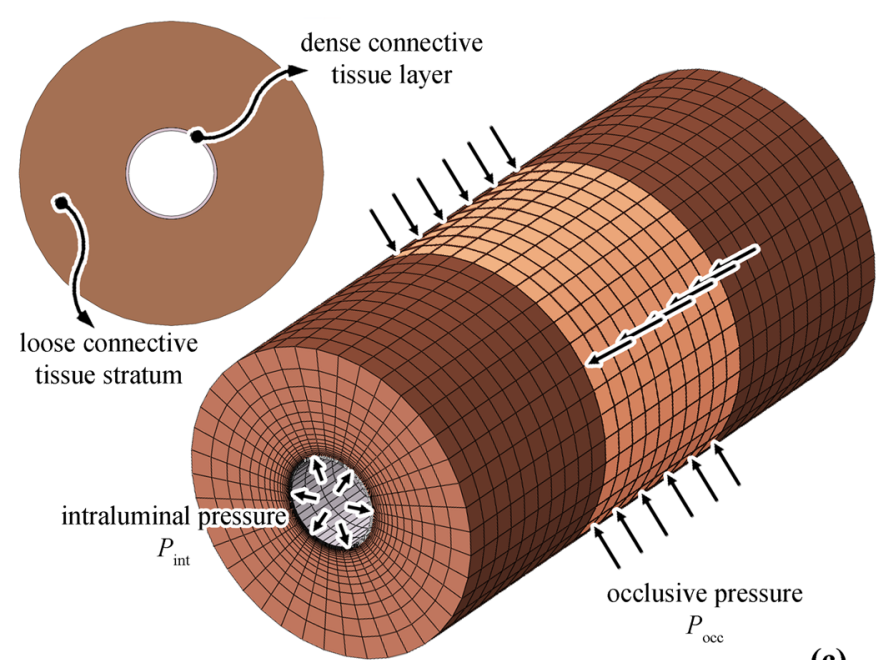

(c)

Fig. 1 Computational models. CFD investigations: virtual solid model of the male lower urinary tract, including bladder and urethra; identification of inlet and outlet regions (a). FSI analyses: virtual solid models of urine fluid domain and urethral wall solid domain, which is characterized by a multi-layered structure; identification of inlet and outlet sections and the region where occlusive pressure is applied (b). CSM simulations: computational model of the urethra

fluid dynamics and solid mechanics has been investigated by the methods of FSI [24]. Due to the computational effort of FSI techniques, the investigation was performed on a $30 \mathrm{~mm}$ length region from bulbar urethra (Fig. 1b). The urethra wall was the solid domain and it was assumed to be composed by a $0.15 \mathrm{~mm}$ thick inner layer of dense connective tissue, as the

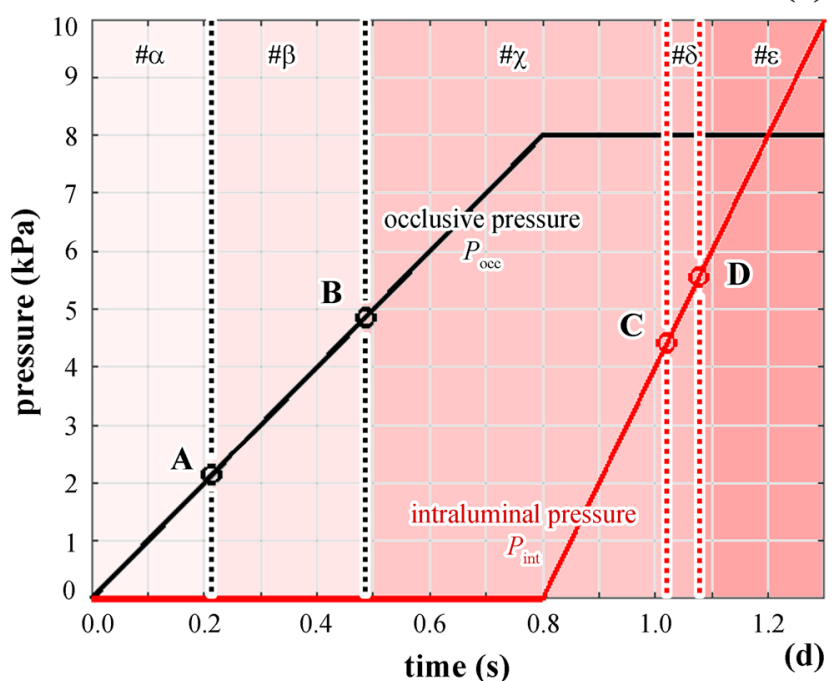

wall and multilayered configuration of the wall; regions of application of the external occlusive pressure and the intraluminal urine pressure (c); time history of occlusive (black line) and intraluminal (red line) pressure. The occlusive pressure increases up to a target value and subsequently the raise of intraluminal pressure begins. Points A, B, C, $D$ denote the transitions between regions $\alpha, \beta, \chi, \delta, \varepsilon(\mathbf{d})$

lamina propria, and a $5.00 \mathrm{~mm}$ thick outer layer of spongy tissue [20]. A fully incompressible Ogden hyperelastic formulation characterized the mechanical behavior of biological tissues [22]. The identification and validation of constitutive parameters of urethral tissues was performed on the basis of data from tensile tests performed on urethral wall samples 
[20]. The inverse analysis of experimental data led to the constitutive parameters of lamina propria and spongy tissue, such as $\mu=20 \mathrm{kPa}$ and $\alpha=6.178, \mu=1.1 \mathrm{kPa}$ and $\alpha=6.178$, respectively [23]. A cylinder with a radius of $2 \mathrm{~mm}$ defined the fluid domain in the undeformed configuration. Urine properties were assumed as reported above for CFD analyses. The geometrical configuration of the system suggested to develop an axis-symmetric model. The solid domain was discretized by 4400 and 9000 rectangular elements for lamina propria and spongy layers. The discretization of the fluid domain assumed rectangular elements within the boundary layer and triangular elements elsewhere (about 30,000 elements). On the lower and the upper boundaries of the solid domain, the displacement along the axial direction was constrained. No slip condition characterized the coupling of solid and fluid domains. The assumption allowed to approximate the continuity of bulbar urethra with the adjacent membranous and penile regions. The fluid boundary conditions aimed to interpret the typical pressure conditions that occur during micturition. Processing results from the previously described CFD analyses provided information about the pressure values along the bulbar urethra. With specific regard to a bladder pressure between 4.0 and $5.0 \mathrm{kPa}$, which is a typical condition that brings to urinate [44], the pressure within the bulbar region is about $3.0 \mathrm{kPa}$, and a variation of at most $0.1 \mathrm{kPa}$ along the assumed $30 \mathrm{~mm}$ length region (Fig. 2a, d) should be expected. Transient FSI simulations were performed. A preliminary step (2 s) aimed to increase the urine pressure from 0 to $2.9 \mathrm{kPa}$ within the entire fluid domain. During the next step, the pressure at the outlet section was kept constant, while at the inlet section the pressure was increased in $2 \mathrm{~s}$ up to a target value, which ranged between 2.9 and $3.0 \mathrm{kPa}$. Afterwards, both inlet and outlet conditions were kept constant for $5 \mathrm{~s}$ aiming to reach an almost steady state flow situation.

FSI analyses have been performed by means of the general purpose code Comsol Multiphysics 5.4 (Comsol Inc., Burlington, MA, USA).

\subsection{Computational Structural Mechanics Analysis of Lumen Occlusion}

The methods of Computational Solid Mechanics allow investigating a further main topic of urology, as the mechanical actions that are required to provide for lumen occlusion. The relevance of the investigation pertains to the design and the setting of AUS devices. A fully three-dimensional model of a $30 \mathrm{~mm}$ length portion of the bulbar urethra was developed (Fig. 1c). The morphometrical configuration and the mechanical behavior of urethral tissues were defined as previously reported in paragraph 2.3. The finite element mesh was developed by means of about 6000 and 20,000 hexahedral elements for lamina propria and spongy layer, respectively. Constraints were imposed on the bottom and the upper surfaces of the cylindrical model aiming to prevent rigid body motions. The occlusive action was defined by means of a radial pressure field that was uniformly applied over a $10 \mathrm{~mm}$ length central region of the outer urethra surface. The action of urine was simulated by means of an intraluminal pressure field that was applied on the lumen surface (Fig. 1c). The developed model was exploited to investigate the relationship between the occlusive action and the intraluminal pressure that is required to open the lumen. The first step of the analysis pertained to lumen occlusion. The intraluminal pressure was null, while the occlusive pressure increased from 0 to the target value. During the next step, the occlusive pressure was kept constant at the target value, while the intraluminal pressure increased from 0 up to $10 \mathrm{kPa}$ (Fig. 1d). The analysis was performed assuming different target values of the occlusive pressure, which ranged between 1 and $12 \mathrm{kPa}$.

The computational analysis was performed by means of the explicit dynamic solver [22] Abaqus Explicit 2018 (Dassault Systèmes, Simulia Corp., Providence, RI, USA).

\subsection{FSI Analysis of Lumen Occlusion}

The CSM investigation of lumen occlusion does not contemplate all the involved physical phenomena. In fact, the action of urine was simulated by means of the pressure it applies on the lumen surface. Aiming to a more reliable investigation, FSI techniques allow to account for the actual fluid behavior of urine. Again, an axis-symmetric model formulation was assumed. The FSI analysis of lumen occlusion and of the subsequent opening because of urine inflow is a real challenge. In fact, the complete occlusion of the lumen should require an unattainable distortion of the fluid domain. Therefore, the FSI investigation was performed by assuming an inverse approach, as the occluding action was imposed after the establishment of the urine flow. The steady state flow condition was provided as described in paragraph 2.3 by assuming a $3 \mathrm{kPa}$ inlet pressure condition. Subsequently, an occlusive pressure field was applied over a $10 \mathrm{~mm}$ length central region of the urethra external surface (Fig. 1b). The occlusive pressure was progressively increased up to the target value in $2 \mathrm{~s}$, and then it was kept constant up to a new steady state condition. Different analyses were performed by assuming different target values of the occlusive pressure, which values were assumed as for CSM analyses. FSI analyses have been performed by means of the general purpose code Comsol Multiphysics 5.4 (Comsol Inc., Burlington, MA, USA).

\section{Results}

Different computational techniques have been exploited aiming to analyze the functionality of the lower urinary tract, with regard to both micturition and lumen occluding actions. 


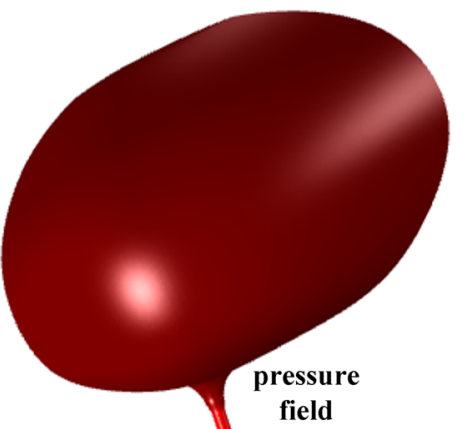

(kPa)
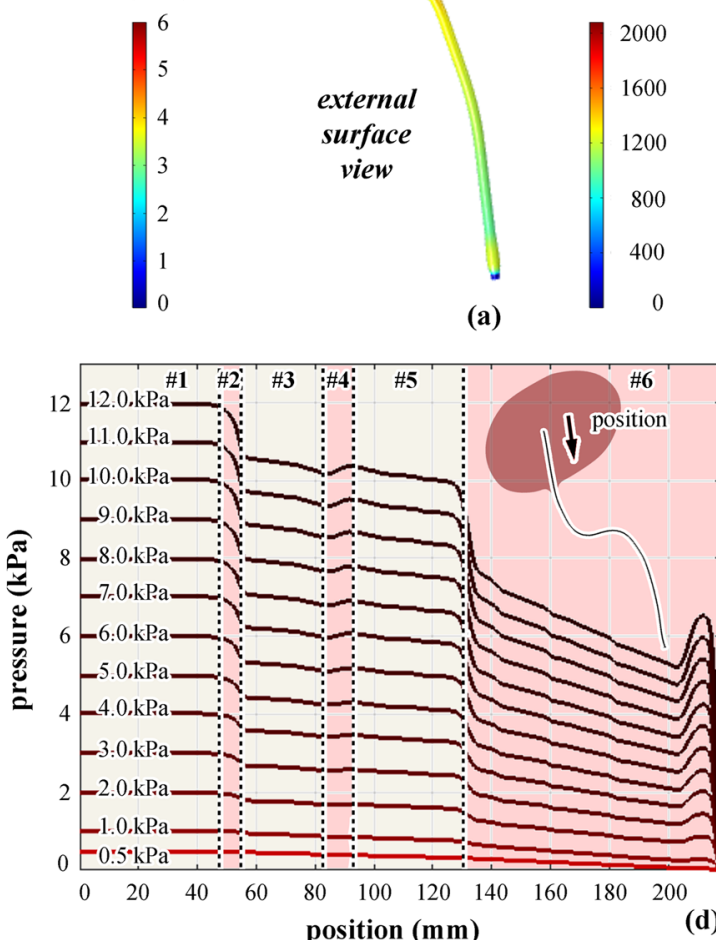

(d)

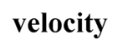
magnitude $(\mathrm{mm} / \mathrm{s})$

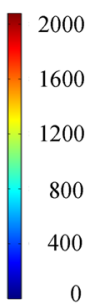

(a)
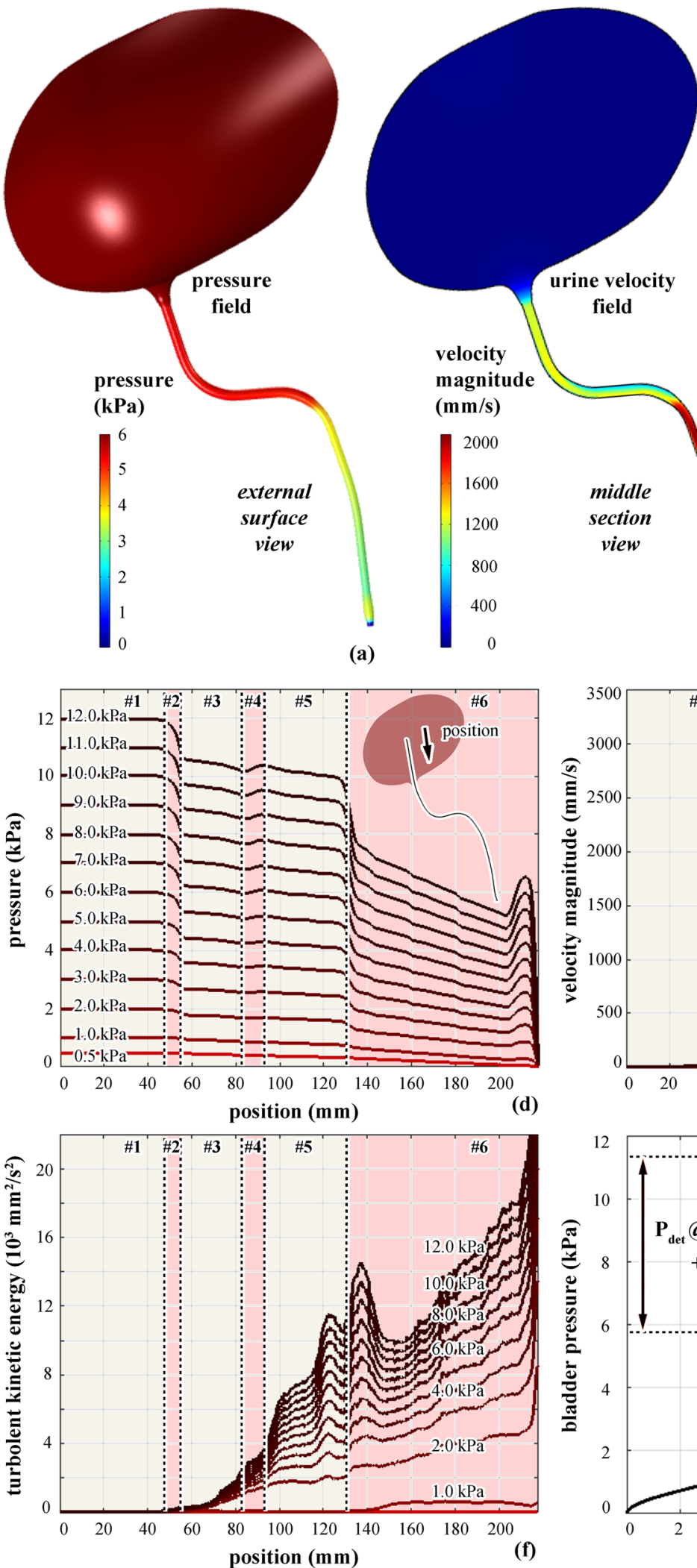

field

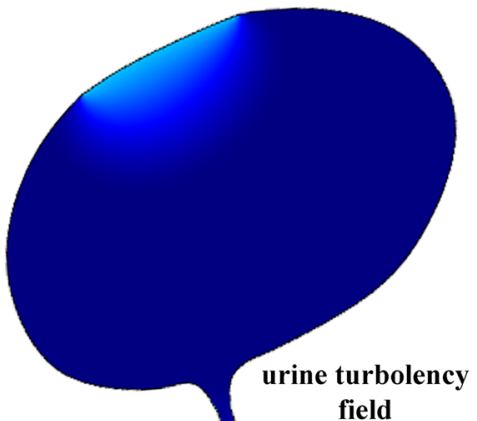

turbolent kinetic energy $\left(\mathrm{mm}^{2} / \mathbf{s}^{2}\right)$

12000
9000
8000
6000
4000
4000
0

(b)
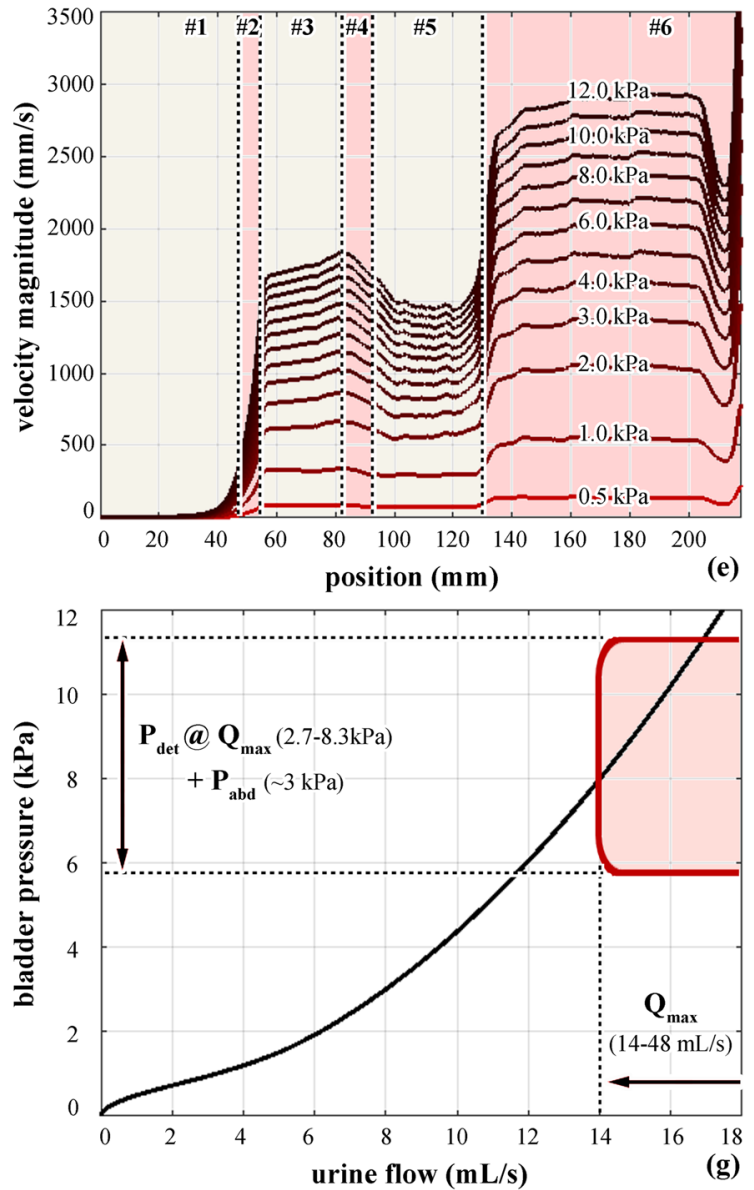
4Fig. 2 CFD investigation of micturition: pressure field at a $6 \mathrm{kPa}$ bladder pressure condition (a); urine velocity field (6 $\mathrm{kPa}$ bladder pressure) (b); turbulent kinetic energy field (6 $\mathrm{kPa}$ bladder pressure) (c); distribution of urine pressure $(\mathbf{d})$, velocity (e) and turbulent kinetic energy (f) along the urethra at different bladder pressure conditions (ranging between 0.5 and $12 \mathrm{kPa}$ ). Relationship between bladder pressure and urine flow: computational results (black line) and average data from clinical measurements (pink rectangle)-bladder pressure accounts for the contributions of both detrusor pressure and abdominal pressure $(\mathbf{g})$

The CFD investigation of urine flow during micturition was performed by assuming a fully $3 \mathrm{D}$ model of both bladder and urethra. The analyses reveled the actual complexity of urine flow. The non-uniformity of lumen dimension and the three-dimensional shape of the urethral duct determine the irregular pressure drop from the bladder to the external meatus (Fig. 2a, d) and the discontinuous changes of urine velocity (Fig. 2b, e). The CFD analysis further allowed to appreciate the occurrence of turbulent phenomena, as described by the turbulent kinetic energy (Fig. 2c, f), which specifies the energy dissipation because of turbulent eddies. Finally, the relationship between bladder pressure and urine flow was analyzed and compared with average data from experimental measurements [43]. Typical urodynamic test usually entails evaluation of detrusor pressure at the maximum urine flow. The ranges of such values were compared with the pressure-flow curve from CFD analysis
(Fig. 2g). Bladder pressure was evaluated by considering the additive contributions of both detrusor pressure and abdominal pressure. Further CFD analyses made it possible to evaluate the influence of urethral strictures on the lower urinary tract functionality, considering both the effects on the pressure-flow relationship (Fig. 3a) and the urine velocity field (Fig. 3b).

Aiming to a more comprehensive investigation of urethral functionality during micturition, the FSI investigation of a urethral segment from the bulbar region was performed. The analyses reveled the actual deformability of the urethra during urine flow, as shown by the displacement field within the urethral wall (Fig. 4a), the relationship between urine flow and the local pressure variation (Fig. 4b) and the profile of urine velocity across the lumen section (Fig. 4c). The investigation further allowed to evaluate the influence of urine flow on the shear stresses that act on the lumen epithelium during micturition (Fig. 4d).

The methods of CSM were exploited aiming to a preliminary investigation of lumen occlusion because of external actions (Fig. 5). The simulation approach allowed to interpret the instability phenomena that the occluding action determines within the urethral wall, and the complex shapes that the lumen assumes (Fig. 5a). The subsequent application of an intraluminal pressure allowed analyzing the effects of urine pressure on the progressive opening of the lumen (Fig. 5b). Results from computational simulations were
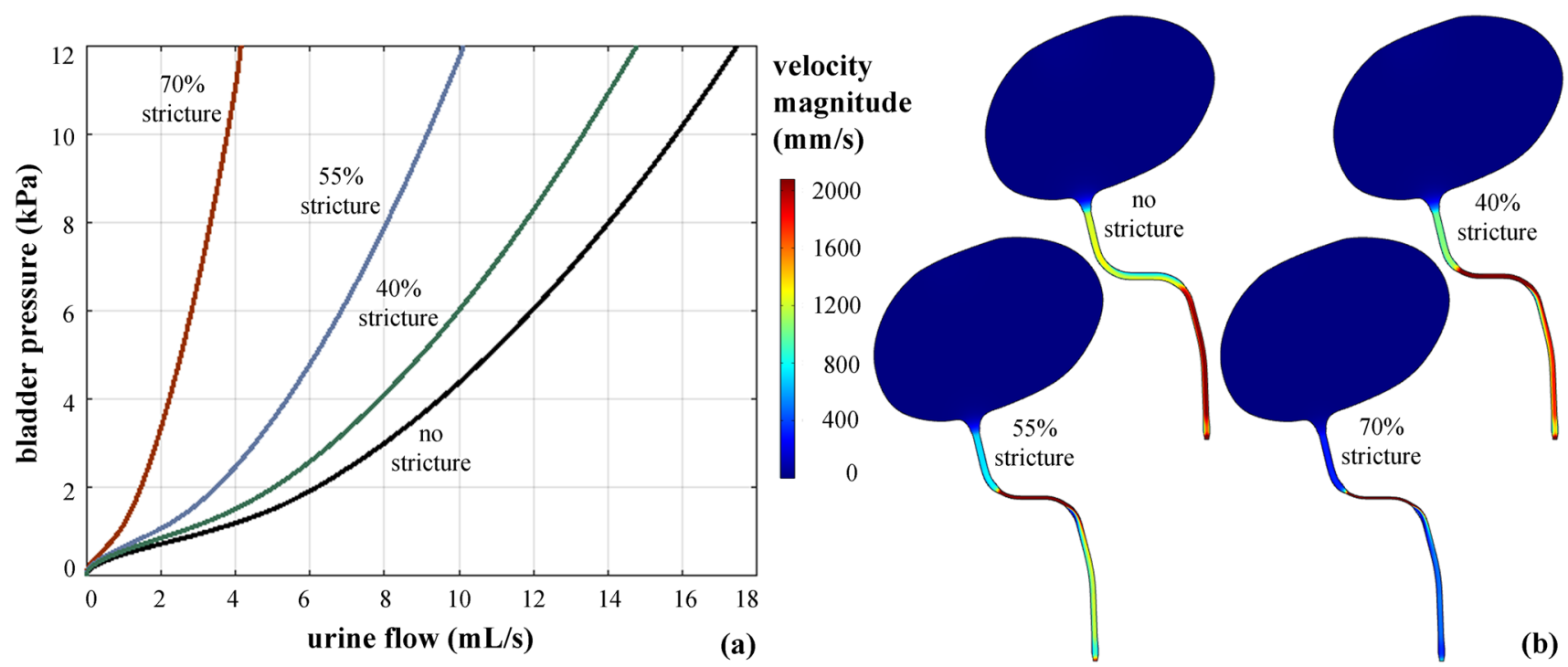

Fig. 3 CFD analysis of the influence of urethral strictures on the lower urinary tract functionality: relationships between bladder pressure and urine flow (a) and urine velocity fields (b) 

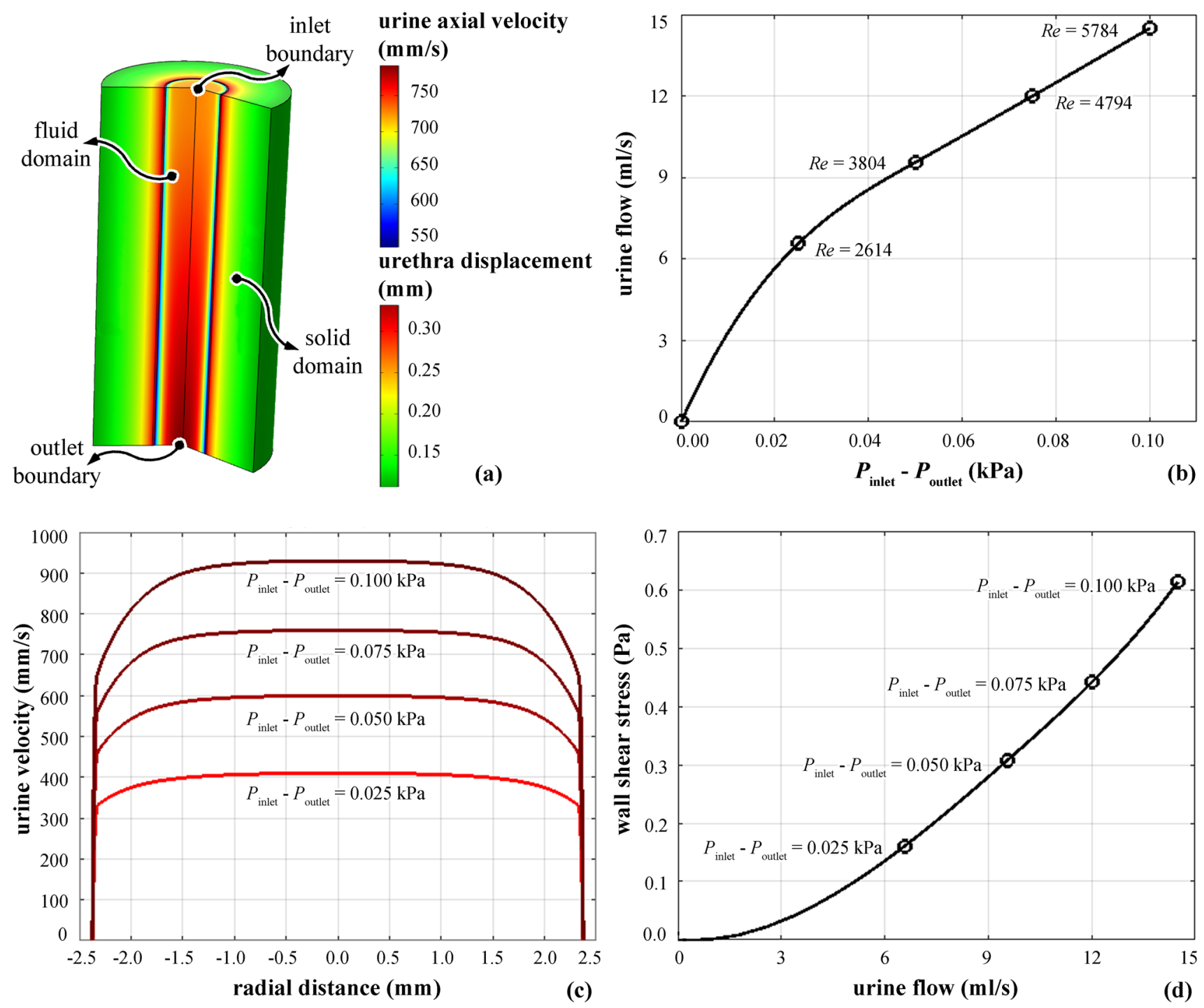

Fig. 4 FSI analysis of micturition within a segment from bulbar urethra: virtual solid models of fluid and solid domains with superposition of urine velocity and wall displacement fields, respectively $(0.1 \mathrm{kPa}$ gradient pressure) (a); trend of pressure variation between the upper and the lower sections of the urethral segment and urine

post-processed aiming to identify the lumen status depending on both occlusive and intraluminal pressure conditions (Fig. 5c). More in details, the progressive increase of occlusive pressure determines, in stage \# $\alpha$ (Figs. 1d, 5a), the continuous reduction of lumen radius. When the occlusive pressure reaches a critical value, the lumen collapses (lumen collapse condition). Instability phenomena implicate a sudden reduction of lumen area and the lumen shapes becomes irregular $[45,46]$. Subsequently, in stage $\# \beta$, the lumen area continuously decreases with the occlusive pressure up the complete lumen occlusion (lumen "zero" area condition). In stage $\# \chi$, the occlusive pressure continues to increase up to the target value and, successively, the application of intraluminal pressure begins. During this stage the lumen

flow, with indication of the corresponding Reynold number condition (b); profile of urine velocity along the lumen diameter at different pressure gradient conditions (c); relationship between urine flow and shear stress acting on the lumen wall (d)

remains occluded. When the intraluminal pressure reaches a critical value, the lumen starts to open and assumes a lenticular shape. The corresponding value of the intraluminal pressure defines the instable opening pressure. The lumen area increases with the intraluminal pressure in stage \# $\#$. When the lumen area returns to the value of the lumen collapse condition, the lumen gets its original circular shape. The corresponding value of the intraluminal pressure defines the stable opening pressure. Subsequently, in stage \# $\varepsilon$, the radius increases with the intraluminal pressure. The specific values of both the stable and the instable opening pressure depend on the applied occlusive pressure. Different analyses were performed to investigate such relationships (Fig. 5d). 

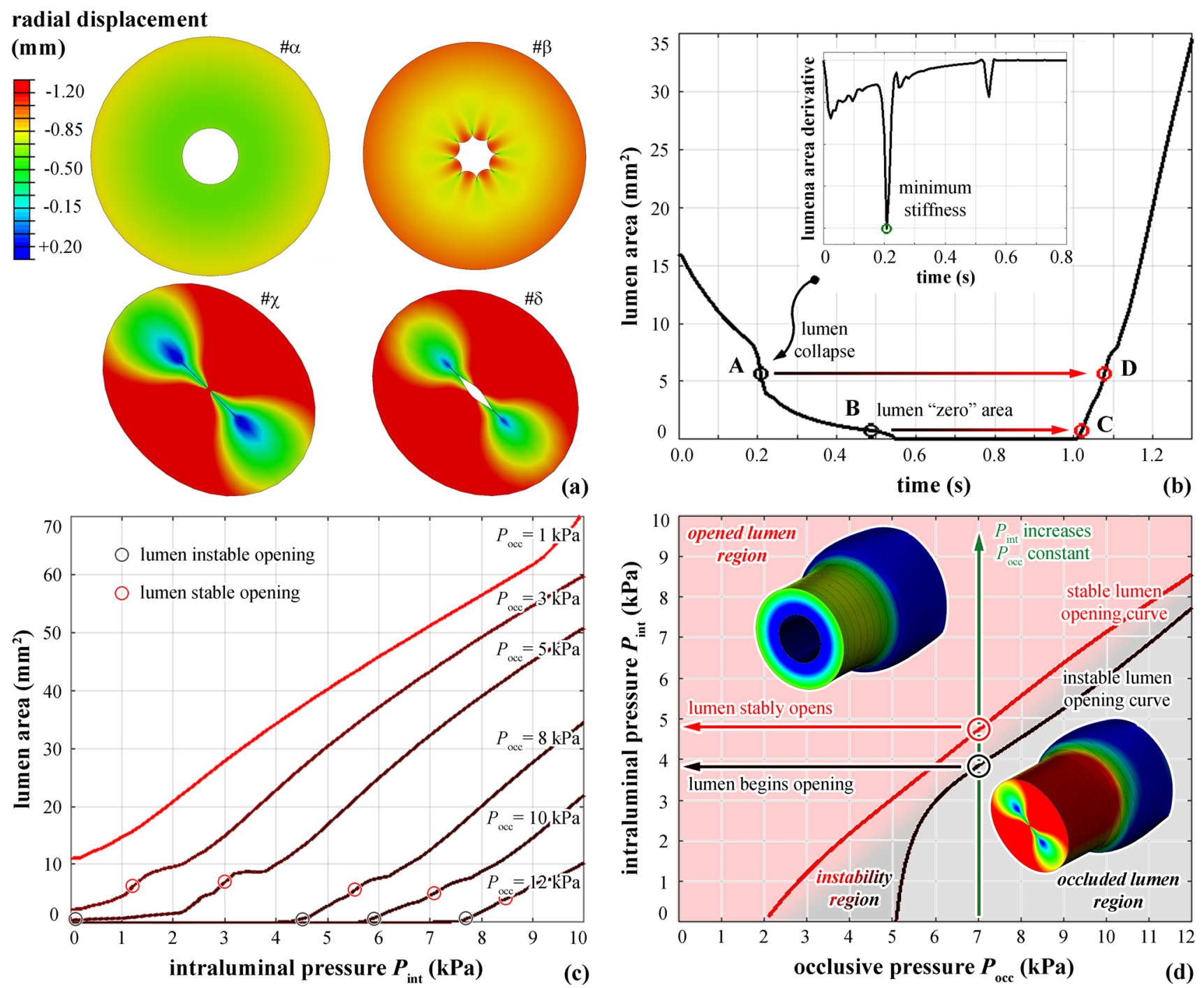

Fig. 5 CSM investigation of urethral lumen occlusion and opening: shape evolution of the urethral section and the lumen during the time history-stable contraction of the lumen (stage $\# \alpha$, as reported in Fig. 1d), instable contraction of the lumen (stage \# $\beta$ ), lumen complete occlusion (stage $\# \chi)$ and lumen instable opening (stage \#ठ) (a); evolution of the lumen area throughout the time history-during stage $\# \alpha$ (before condition A) the lumen shape is regular and its area continuously decreases with the occluding pressure up to a critical condition (lumen collapse condition), when the area suddenly falls (the derivative of lumen area reaches a minimum); during stage $\# \beta$ (between points $\mathrm{A}$ and $\mathrm{B}$ ) the lumen shape seems indented and its area continues to decrease up to lumen closure (lumen "zero" area condition); during stage $\# \chi$ (between point $B$ and $C$ ) the lumen is occluded,

Finally, FSI techniques made it possible to investigate the comprehensive fluid-mechanical functionality of the urethra accounting for the influence of external occluding actions. Given a specific bladder pressure condition, the developed analyses provided data about the actual influence of the occlusive pressure on lumen dimension (Fig. 6a), urine flow

the occlusive pressure reaches the target value and the intraluminal pressure increases; stage \# $\delta$ (between points $C$ and $D$ ) starts when the lumen begins to open according to a lenticular shape; stage \# lasts until the lumen returns to a stable shape; during stage \# $\varepsilon$ (after point $\mathrm{D}$ ) the lumen shape is regular again and its area continuously increases with intraluminal pressure (b); evaluation of the relationship between lumen area and intraluminal pressure for different target values of the occlusive pressure (c); plot of the lumen status depending on both occlusive pressure and intraluminal pressure conditions the lumen is stably opened within the upper-left region of the graph, while it is occluded within the bottom-right region; the two portions of the pressure map are separated by the instability region, where the lumen shape appears indented or lenticular (d)

(Fig. 6b) and urine velocity profile (Fig. 6c). The investigation further allowed to evaluate the effects of occluding actions on flow turbulence (Fig. 6d), actual urine pressure in the occluded region (Fig. 6e) and shear stress acting on the lumen wall (Fig. 6f). 

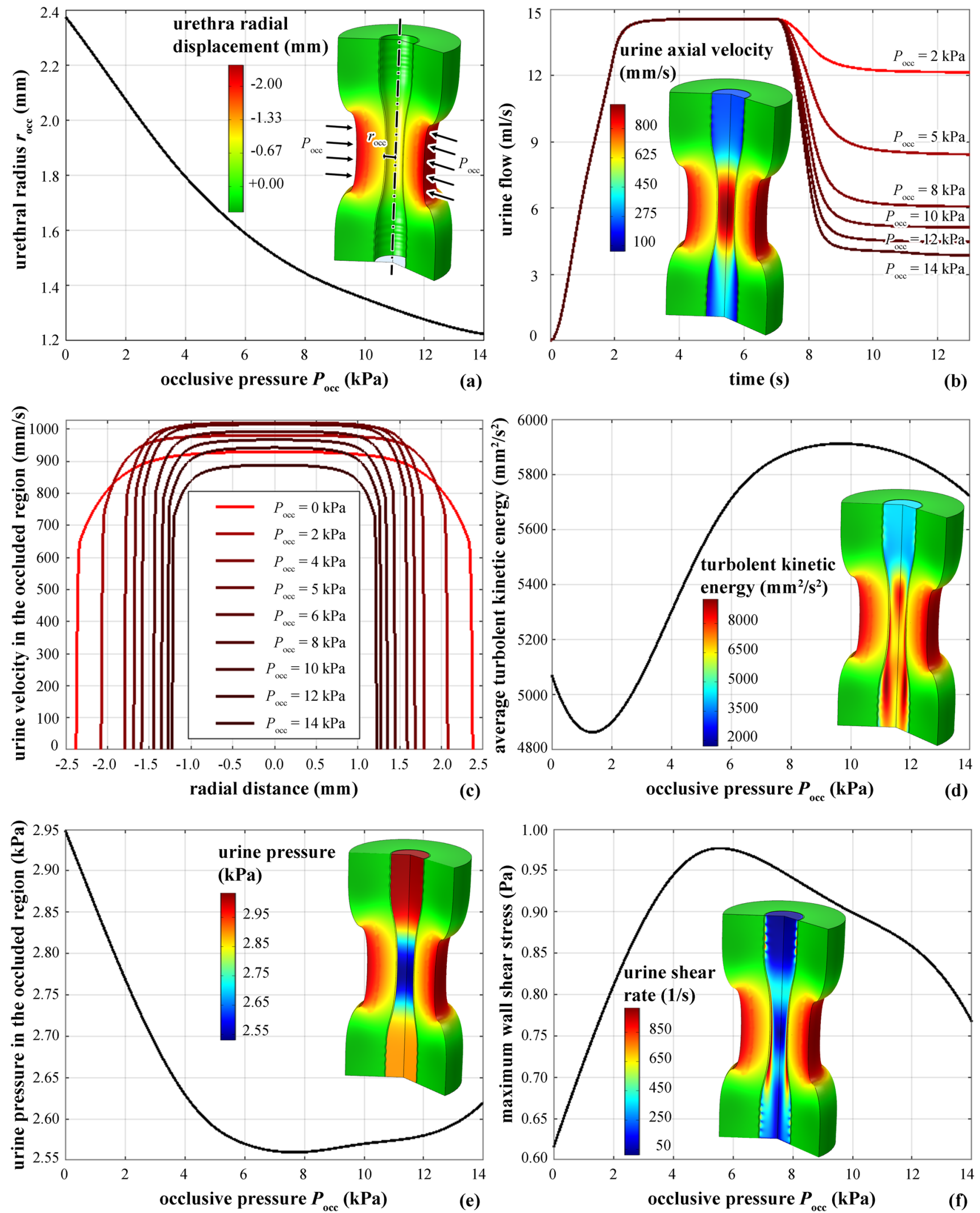
४Fig. 6 Fluid Structure Interaction analysis of urethral lumen occlusion during micturition at a $0.1 \mathrm{kPa}$ gradient pressure condition: relationship between lumen radius and occlusive pressure (a); time history of urine flow at different occlusive pressure conditions- the urine flow progressively increases with the pressure gradient $(0-2 \mathrm{~s})$; the pressure gradient is kept constant and the urine flow reaches a steady state condition ( $2-7 \mathrm{~s})$; the occlusive pressure is progressively applied up to the target value and the urine flow decreases (7-9 s); the occlusive pressure is kept constant and the urine flow reaches a new steady state condition (9-13 s) (b); profile of urine velocity along the lumen diameter at different occlusive pressure conditions (c); relationship between occlusive pressure energy dissipation because of turbulent phenomena (d), urine pressure in the occluded region (e), and maximum shear stress at the lumen wall (f)

\section{Discussion and Conclusions}

The reported models, analyses and results proved the reliability and effectiveness of computational methods for the investigation of the lower urinary tract functionality and physiology in health and in disease. CFD investigations of micturition highlight the actual complexity of urine flow throughout the urethra. The anatomical configuration of the urethra entails the nonlinear pressure drop from bladder to the urinary meatus (Fig. 2a, d) and the non-homogenous distribution of urine velocity (Figs. 2b, e). Both inlet pressure conditions and complex geometrical conformation entail turbulent phenomena, with particular regard to the bulbar region and the urinary meatus. The CFD approach further allows analyzing the effects of urethral obstructions, such in the case of urethral strictures (Fig. 3). Such information are complementary with data from urodynamic clinical investigations, providing detailed descriptions of urine flow parameters, such as the pressure-flow trend (Fig. 3a) and the urine velocity field (Fig. 3b), which can be correlated with pathologies and diseases. Computational results highlight the significant influence of urethral strictures on flow resistance, as it is reported by the clinical experience [47].

Previous CFD investigations reported about the influence of urethral strictures on the urine flow field assuming laminar flow conditions [17]. On the other side, turbulent phenomena may characterize urine flow, as previously reported by means of 2D CFD models [15]. The here proposed CFD approach accounts for both the actual 3D conformation of the lower urinary tract and turbulent phenomena. FSI investigations further prove the arising of urine turbulences within the bulbar region, because Reynolds numbers greater than 2000 characterize the local flow [13] (Fig. 4b). The investigation and the prediction of turbulences is a relevant topic, because turbulent urinary flow in the urethra may be a causal factor for different pathologies and diseases, as prostatic hyperplasia [48], reflux of urine in the prostatic ducts, prostatitis $[49,50]$ and urinary tract infections, such as dysuria [51]. Furthermore CFD and FSI techniques allow the quantitative identification of the shear stresses that urine flow plays on the lumen wall (Figs. 4d and 6f). Shear stresses stimulate the epithelium and the local urethral-neuroendocrine cells. Such cells release mediators and, via activation of adjacent sensory nerves, stimulate urethral reflexes [52] and influence continence and sensation [53]. Furthermore, many investigations have been reported in the field of vascular fluid-dynamics about the role of shear stresses on endothelial cells physiology [54]. Shear stresses induce morphological changes of endothelium and vessel wall, but also trigger biochemical and biological events. There is considerable evidence that physiologic stresses exert vasoprotective roles and a perturbation of the stresses can lead to remodeling and possible dysfunction [55]. Similar phenomena may characterize the urethral epithelium. It further follows the relevance of investigating the influence of occlusive pressure on wall shear stresses. With regard to AUS patients, nevertheless the device is deactivated during micturition, it anyway exerts mechanical actions on the urethra, which lead to a magnification of shear stresses (Fig. 6f). With specific regard to the lower urinary tract, previous FSI investigations were performed to analyze the ureteral pressure-flow relationship, considering also the influence of occlusive actions [5]. The here proposed analyses highlight the relevance of the FSI approach for the investigation of urethra functionality considering both the ordinary micturition condition and the occlusion by AUS devices.

The reliability of the developed models is assessed by the mutual comparison of results and the evaluation of data from experimental activities and clinical tests. As an example, the CFD model prediction of the relationship between bladder pressure and urine flow well interpret results from urological tests (Figs. 2g, 3a) [47]. The urine flow predictions from both CFD and FSI analyses provide comparable results at corresponding local pressure conditions (Figs. 2d, $\mathrm{g}$ and $4 \mathrm{~b}$ ). The CSM predictions of occlusive pressure values that ensure lumen occlusion are comparable with clinical data from AUS surgery [26, 56].

The here proposed models just provide a preliminary approach to the computational investigation of the lower urinary tract. A more refined investigation of urine flow during micturition should require a FSI model of both bladder and urethra. Furthermore, the modeling of the lumen shape should be performed accounting for its actual complex morphology. Such a model should be exploited aiming to analyze many different diseases, as urethra strictures or prostatic obstructions [17]. With regard to the investigation of lumen occlusion mechanisms by AUS devices, the modeling of the prosthetic device and of its interaction with the urethra is mandatory. This is part of further analyses that are under development at the University of Padova [32]. 
Funding Open Access funding provided by Università degli Studi di Padova. This study has been partially supported by University of Padova, Project FONT_BIRD2020_01 "Characterization of Artificial Urinary Sphincters for the identification of new DEVices (AUS-DEV)".

Data Availability The models, which have been described in the paper and exploited to achieve the provided results, have been deposited within the following dataset: https://datadryad.org/stash/share/SqRj2 waxWHtI3boO0moMww6e35ZoAtEvrYDCagqWC4E

\section{Compliance with Ethical Standards}

Conflict of interest The author declares that he has no conflict of interest.

Ethical Approval The author has seen and approved the final version of the manuscript. The author warrants that the article is the authors' original work, hasn't received prior publication and isn't under consideration for publication elsewhere.

Open Access This article is licensed under a Creative Commons Attribution 4.0 International License, which permits use, sharing, adaptation, distribution and reproduction in any medium or format, as long as you give appropriate credit to the original author(s) and the source, provide a link to the Creative Commons licence, and indicate if changes were made. The images or other third party material in this article are included in the article's Creative Commons licence, unless indicated otherwise in a credit line to the material. If material is not included in the article's Creative Commons licence and your intended use is not permitted by statutory regulation or exceeds the permitted use, you will need to obtain permission directly from the copyright holder. To view a copy of this licence, visit http://creativecommons.org/licenses/by/4.0/.

\section{References}

1. Stibitz, G. R. (1965). A mathematical model of the urethra. Bulletin of Mathematical Biophysics., 27, 407-415.

2. Griffiths, D. J. (1973). The mechanics of the urethra and of micturition. British Journal of Urology, 45(5), 497-507.

3. Valentini, F. A., Besson, G. R., Nelson, P. P., \& Zimmern, P. E. (2000). A mathematical micturition model to restore simple flow recordings in healthy and symptomatic individuals and enhance uroflow interpretation. Neurourology and Urodynamics, 19(2), 153-176.

4. Paya, A. S., Fernandez, D. R., Gil, D., Garcia Chamizo, J. M., \& Perez, F. M. (2013). Mathematical modelling of the lower urinary tract. Computer Methods and Programs in Biomedicine, 109(3), 323-338.

5. Vahidi, B., Fatouraee, N., Imanparast, A., et al. (2011). A mathematical simulation of the ureter: Effects of the model parameters on ureteral pressure/flow relations. Journal of Biomechanical Engineering, 133(3), 031004.

6. Rudyk, R., Malinowski, M., Mackiewicz, A., Bedzinski, R., Noszczyk-Nowak, A., Skonieczna, J., \& Madej, J. (2020). Numerical analysis of deformation and flow in the proximal area of the urethra. International Journal of Applied Mechanics and Engineering, 25, 130-141.

7. Mahadevan, V. (2016). Anatomy of the lower urinary tract. Surgery $($ Oxford $)$., 34(7), 318-325.

8. Lierse, W. (1987). Male urethra. Applied anatomy of the pelvis. Berlin: Springer.
9. Pullan, B. R., Phillips, J. I., \& Hickey, D. S. (1982). Urethral lumen cross-sectional shape: Its radiological determination and relationship to function. BJU International, 54, 399-407.

10. Brandes, B., \& Morey, A. F. (2014). Advanced male urethral and genital reconstructive surgery (2nd ed.). Berlin: Springer.

11. Drake, M. J., Doumouchtsis, S. K., Hashim, H., \& Gammie, A. (2018). Fundamentals of urodynamic practice, based on International Continence Society good urodynamic practices recommendations. Neurourology and Urodynamics, 37(S6), S50-S60.

12. Zeng, R. (2020). Dysuria. In X. H. Wan \& R. Zeng (Eds.), Handbook of clinical diagnostics. Singapore: Springer.

13. Moukalled, F., Mangani, L., Darwish, M. (2016) The finite volume method in computational fluid dynamics. Berlin: Springer.

14. Jin, Q., Zhang, X., Li, X., et al. (2010). Dynamics analysis of bladder-urethra system based on CFD. Frontiers of Mechanical Engineering in China, 5, 336-340.

15. Pel, J. J., \& van Mastrigt, R. (2007). Development of a CFD urethral model to study flow-generated vortices under different conditions of prostatic obstruction. Physiological Measurement, 28(1), $13-23$.

16. Ishii, T., Naya, Y., Yamanishi, T., \& Igarashi, T. (2014). Urine flow dynamics through the urethra in patients with bladder outlet obstruction. Journal of Mechanics in Medicine and Biology, 14(4), 1450052.

17. Cohen, A. J., Baradaran, N., Mena, J., et al. (2019). Computational fluid dynamic modeling of urethral strictures. Journal of Urology, 202(2), 347-353.

18. Bremnor, J. D., \& Sadovsky, R. (2002). Evaluation of dysuria in adults. American Family Physician, 65(8), 1589-1596.

19. Michels, T. C., \& Sands, J. E. (2015). Dysuria: Evaluation and differential diagnosis in adults. American Family Physician, 92(9), 778-786.

20. Natali, A. N., Carniel, E. L., Frigo, A., et al. (2016). Experimental investigation of the biomechanics of urethral tissues and structures. Experimental Physiology, 101(5), 641-656.

21. Masri, C., Chagnon, G., Favier, D., Sartelet, H., \& Girard, E. (2018). Experimental characterization and constitutive modeling of the biomechanical behavior of male human urethral tissues validated by histological observations. Biomechanics and Modeling in Mechanobiology, 17(4), 939-950.

22. Belytschko, T., Liu, W. K., Moran, B., et al. (2013). Nonlinear finite elements for continua and structures (2nd ed.). New York: Wiley.

23. Natali, A. N., Carniel, E. L., Fontanella, C. G., et al. (2017). Mechanics of the urethral duct: Tissue constitutive formulation and structural modeling for the investigation of lumen occlusion. Biomechanics and Modeling in Mechanobiology, 16(2), 439-447.

24. Hron, J., \& Mádlík, M. (2007). Fluid-structure interaction with applications in biomechanics. Nonlinear Analysis: Real World Applications, 8(5), 1431-1458.

25. Scotti, C. M., Shkolnik, A. D., Muluk, S. C., et al. (2005). Fluidstructure interaction in abdominal aortic aneurysms: Effects of asymmetry and wall thickness. BioMedical Engineering Online, 4, 64.

26. Marziale, L., Lucarini, G., Mazzocchi, T., Gruppioni, E., Castellano, S., Davalli, A., et al. (2018). Artificial sphincters to manage urinary incontinence: A review. Artificial Organs, 42(9), E215-E233.

27. Guralnick, M. L., Miller, E., Toh, K. L., et al. (2002). Transcorporal artificial urinary sphincter cuff placement in cases requiring revision for erosion and urethral atrophy. Journal of Urology, 167(5), 2075-2079.

28. Srivastava, A., Joice, G. A., Patel, H. D., Manka, M. G., Sopko, N. A., \& Wright, E. J. (2019). Causes of artificial urinary sphincter failure and strategies for surgical revision: Implications of device component survival. European Urology Focus, 5(5), 887-893. 
29. Carson, C. C. (2020). Artificial urinary sphincter: Current status and future directions. Asian Journal of Andrology, 22(2), $154-157$.

30. Ostrowski, I., Śledź, E., Wilamowski, J., Józefczak, M., Dyś, W., Ciechan, J., et al. (2020). Patients' quality of life after implantation of ZSI 375 artificial urinary sphincter due to stress urinary incontinence. Central European Journal of Urology, 73(2), 178-186.

31. Natali, A. N., Carniel, E. L., \& Fontanella, C. G. (2019). Interaction phenomena between a cuff of an artificial urinary sphincter and a urethral phantom. Artificial Organs, 43(9), 888-896.

32. Natali, A. N., Fontanella, C. G., \& Carniel, E. L. (2020). Biomechanical analysis of the interaction phenomena between artificial urinary sphincter and urethral duct. International Journal for Numerical Methods in Biomedical Engineering, 36(3), e3308.

33. Natali, A. N., Carniel, E. L., \& Fontanella, C. G. (2020). Investigation of interaction phenomena between lower urinary tract and artificial urinary sphincter in consideration of urethral tissues degeneration. Biomechanics and Modeling in Mechanobiology, 19(6), 2099-2109.

34. Marziale, L., Lucarini, G., Mazzocchi, T., Ricotti, L., \& Menciassi, A. (2020). Comparative analysis of occlusion methods for artificial sphincters. Artificial Organs. https://doi.org/10.1111/ aor.13684.

35. Korossis, S., Bolland, F., Southgate, J., et al. (2009). Regional biomechanical and histological characterisation of the passive porcine urinary bladder: Implications for augmentation and tissue engineering strategies. Biomaterials, 30(2), 266-275.

36. Gloeckner, D. C., Sacks, M. S., Fraser, M. O., et al. (2002). Passive biaxial mechanical properties of the rat bladder wall after spinal cord injury. Journal of Urology, 167(5), 2247-2252.

37. Natali, A. N., Carniel, E. L., Frigo, A., Fontanella, C. G., Rubini, A., Avital, Y., \& De Benedictis, G. M. (2017). Experimental investigation of the structural behavior of equine urethra. Computer Methods and Programs in Biomedicine, 141, 35-41.

38. Davis, N. F., Cunnane, E. M., Mooney, R. O., et al. (2018). Characterisation of human urethral rupture thresholds for urinary catheter inflation related injuries. Journal of the Mechanical Behavior of Biomedical Materials, 83, 102-107.

39. Inman, B. A., Etienne, W., Rubin, R., et al. (2013). The impact of temperature and urinary constituents on urine viscosity and its relevance to bladder hyperthermia treatment. Int J Hyperthermia., 29(3), 206-210.

40. Fusco, M. A., Martin, R. S., \& Chang, M. C. (2001). Estimation of intra-abdominal pressure by bladder pressure measurement: Validity and methodology. Journal of Trauma, 50(2), 297-302.

41. van Mastrigt, R., Huang, F., \& Chung, J. W. (2006). Bladder volume sensitivity of isovolumetric intravesical pressure. Neurourology and Urodynamics, 25(7), 744-751.

42. Clausen, I., Tvedt, W. L. G., \& Glott, T. (2018). Measurement of urinary bladder pressure: A comparison of methods. Sensors (Basel), 18(7), 2128.
43. Nitti, V. W. (2005). Pressure flow urodynamic studies: The gold standard for diagnosing bladder outlet obstruction. Review of Urology, 7(Suppl 6), S14-21.

44. Houle, A. M., Gilmour, R. F., Churchill, B. M., Gaumond, M., \& Bissonnette, B. (1993). What volume can a child normally store in the bladder at a safe pressure? Journal of Urology, 149(3), 561-564.

45. Zhu, Y., Luo, X. Y., Wang, H. M., Ogden, R. W., \& Berry, C. (2013). Three-dimensional non-linear buckling of thick-walled elastic tubes under pressure. International Journal of Non-Linear Mechanics., 48, 1-14.

46. Kozlovsky, P., Zaretsky, U., Jaffa, A. J., \& Elad, D. (2014). General tube law for collapsible thin and thick-wall tubes. Journal of Biomechanics, 47(10), 2378-2384.

47. Sekido, N. (2012). Bladder contractility and urethral resistance relation: What does a pressure flow study tell us? International Journal of Urology, 19, 216-228.

48. Martinez-Borges, A. R. (2006). Turbulent urinary flow in the urethra could be a causal factor for benign prostatic hyperplasia. Medical Hypotheses, 67(4), 871-875.

49. Caropreso, D., \& Moon, T. D. (2000). Alpha-blockers: An effective treatment for prostatitis? Current Urology Reports, 1(2), $148-154$.

50. Genao, L., \& Buhr, G. T. (2012). Urinary tract infections in older adults residing in long-term care facilities. The Annals of LongTerm Care, 20(4), 33-38.

51. Vigil, H. R., \& Hickling, D. R. (2016). Urinary tract infection in the neurogenic bladder. Translational Andrology and Urology, 5(1), 72-87.

52. Birder, L. A., Andersson, K. E., Kanai, A. J., et al. (2014). Urothelial mucosal signaling and the overactive bladder-ICI-RS 2013. Neurourology and Urodynamics, 33(5), 597-601.

53. Khandelwal, P., Abraham, S. N., \& Apodaca, G. (2009). Cell biology and physiology of the uroepithelium. American Journal of Physiology, Renal Physiology, 297(6), F1477-F1501.

54. Paszkowiak, J. J., \& Dardik, A. (2003). Arterial wall shear stress: Observations from the bench to the bedside. Vascular and Endovascular Surgery, 37(1), 47-57.

55. Lu, D., \& Kassab, G. S. (2011). Role of shear stress and stretch in vascular mechanobiology. Journal of the Royal Society, Interface, 8(63), 1379-1385.

56. Cordon, B. H., Singla, N., \& Singla, A. K. (2016). Artificial urinary sphincters for male stress urinary incontinence: Current perspectives. Medical Devices (Auckland)., 9, 175-183.

57. Clement, K. D., Burden, H., Warren, K., et al. (2015). Invasive urodynamic studies for the management of lower urinary tract symptoms (LUTS) in men with voiding dysfunction. Cochrane Database of Systematic Reviews, 4, CD011179.

58. Bugeja, S., Ivaz, S. L., Frost, A., et al. (2016). Urethral atrophy after implantation of an artificial urinary sphincter: Fact or fiction? BJU International, 117(4), 669-676. 